Please do not remove this page

RMIT

UNIVERSITY

\title{
Automated ATM system enabling 4DT-based operations
}

Gardi, Alessandro Giacomo Maria; Sabatini, Roberto; Ramasamy, Subramanian; Marino, Matthew; Kistan, Trevor

https://researchrepository.rmit.edu.au/esploro/outputs/9921862454201341/filesAndLinks?institution=61RMIT_INST\&index=null

Gardi, A. G. M., Sabatini, R., Ramasamy, S., Marino, M., \& Kistan, T. (2015). Automated ATM system enabling 4DT-based operations. Proceedings of the SAE 2015 AeroTech Congress and Exhibition, 1-7. https://doi.org/10.4271/2015-01-2539

Document Version: Accepted Manuscript

Published Version: https://doi.org/10.4271/2015-01-2539

Repository homepage: https://researchrepository.rmit.edu.au

Copyright ( 2015 SAE International

Downloaded On 2023/04/26 21:04:12 +1000

Please do not remove this page 
Thank you for downloading this document from the RMIT Research Repository.

The RMIT Research Repository is an open access database showcasing the research outputs of RMIT University researchers.

RMIT Research Repository: http://researchbank.rmit.edu.au/

\section{Citation:}

Gardi, A, Sabatini, R, Ramasamy, S, Marino, M and Kistan, T 2015, 'Automated ATM system enabling 4DT-based operations', in Proceedings of the SAE 2015 AeroTech

Congress and Exhibition, Warrendale, PA, United States, 22-24 September 2015, pp. 1-7.

See this record in the RMIT Research Repository at:

https://researchbank.rmit.edu.au/view/rmit:33430

Version: Accepted Manuscript

Copyright Statement: (C) 2015 SAE International

Link to Published Version:

http://dx.doi.org/10.4271/2015-01-2539 


\title{
Automated ATM System Enabling 4DT-Based Operations
}

\author{
Alessandro Gardi, Roberto Sabatini, Subramanian Ramasamy, Matthew Marino, Trevor Kistan \\ RMIT University - SAMME, Melbourne, Australia
}

\begin{abstract}
As part of the current initiatives aimed at enhancing safety, efficiency and environmental sustainability of aviation, a significant improvement in the efficiency of aircraft operations is currently pursued. Innovative Communication, Navigation, Surveillance and Air Traffic Management (CNS/ATM) technologies and operational concepts are being developed to achieve the ambitious goals for efficiency and environmental sustainability set by national and international aviation organizations. These technological and operational innovations will be ultimately enabled by the introduction of novel CNS/ATM and Avionics (CNS+A) systems, featuring higher levels of automation. A core feature of such systems consists in the real-time multi-objective optimization of flight trajectories, incorporating all the operational, economic and environmental aspects of the aircraft mission. This article describes the conceptual design of an innovative ground-based Air Traffic Management (ATM) system featuring automated 4-Dimensional Trajectory (4DT) functionalities. The 4DT planning capability is based on the multi-objective optimization of 4DT intents. After summarizing the concept of operations, the top-level system architecture and the key 4DT optimization modules, we discuss the segmentation algorithm to obtain flyable and concisely described 4DT. Simulation case studies in representative scenarios show that the adopted algorithms generate solutions consistently within the timeframe of online tactical rerouting tasks, meeting the set design requirements.
\end{abstract}

\section{Introduction}

Major air transport modernisation initiatives including the Next Generation Air Transportation System (NextGen) in the US, the Single European Sky Air Traffic Management Research (SESAR), and Clean Sky Joint Technology Initiative for Aeronautics and Air Transport in Europe were established to improve the operational efficiency, safety and environmental sustainability of aviation. In order to achieve the ambitious objectives set by national and international organizations for the future in an increasing traffic growth trend, it will be essential to fully exploit the most advanced technologies and develop new enhanced functionalities specifically tailored for the online Air Traffic Management (ATM) domain. The air traffic, in fact, is subject to a substantial number of unpredicted events and perturbations, which often lead to congestions and delays at the destination airport, or to large-scale re-routings, significantly deteriorating most of the environmental and economic performances. In this perspective, a number of earlier studies identified the opportunity of introducing increasing levels of automation and discussed the development of automated ATM systems [1-5]. The adoption of 4-Dimensional Trajectories (4DT) in an Intent Based Operations (IBO) environment has been identified as the key step for the enhanced exploitation of the available airspace resources, mitigating the effects of unpredicted disruptions. Novel Communications, Navigation, Surveillance, ATM (CNS/ATM) and Avionics (CNS+A) systems are currently being developed to enable the 4DT-IBO. These systems will incorporate algorithms for the online generation, validation, execution and monitoring of optimized 4DT intents. Although the optimization of flight trajectories has been studied for a significant time and a number of numerical solution strategies have been proposed, substantial practical gains are still unattained due to the limitations of the currently employed air traffic and airspace models. The dynamics of the air traffic, airspace and atmosphere have to be fully considered and implemented in the algorithms systems in order to significantly enhance the predictability and the resilience to perturbations, and consequently extend the validity and accuracy of 4DT planning. This paper expand the development of the ground-based 4DT Planning, Negotiation and Validation (4-PNV) system [6-9], which is a substantially innovative evolution intended to introduce online 4DT management functionalities including a multi-objective trajectory optimization algorithm.

\section{CNS+A Concept of Operations}

The CNS+A concept of operations in the online 4DT-IBO context is schematically represented in Fig. 1.

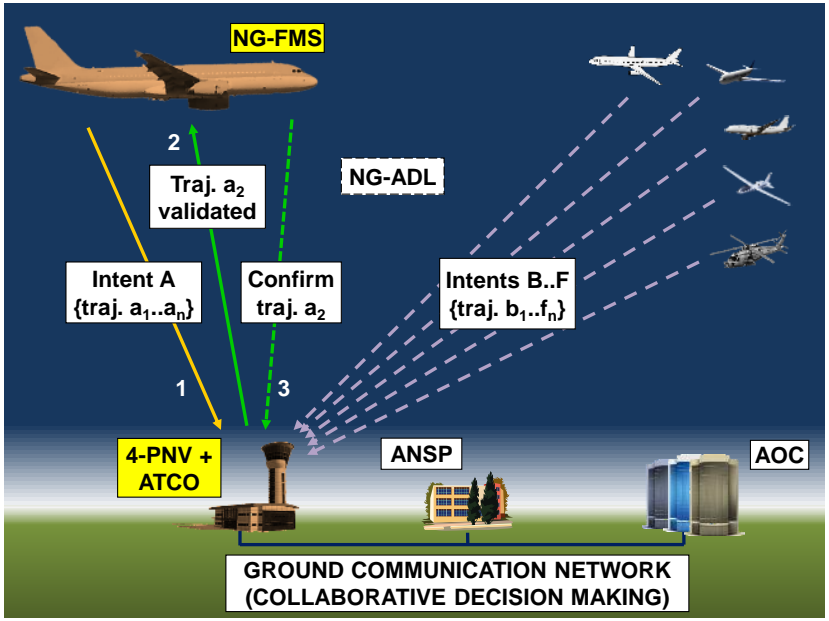

Figure 1. Online CNS+A concept of operations. 
The 4-PNV system features 4DT planning, data exchange and negotiation/validation with the Next Generation of Flight Management Systems (NG-FMS) on board manned aircraft and Mission Management Systems (NG-MMS) on board RPAS [9]. The optimized 4DT intents generated by the NG-FMS and NGMMS are downlinked to the ground-based 4-PNV system via a Next Generation Aeronautical Data-Link (NG-ADL). The NGADL considered here is a further step in the evolution of the datalinks for civil aviation that are currently being enhanced to fulfill the requirements set within SESAR/NextGen. Several 4DT intents are generated by each NG-FMS/MMS, providing multiple options to identify conflict free solutions in real-time [6], hence decreasing the negotiation timespans and reducing the need for remote 4DT generation by ATM. The 4-PNV analyses and identifies the globally optimal combination of intents to best achieve global objectives, whilst ensuring that the validated 4DT are free from traffic conflicts, weather hazards and congested sectors. Once one of the identified optimal and valid 4DT has been reviewed and approved by the human Air Traffic Control Operators (ATCO), the 4DT validation is accomplished and an ATM clearance is uplinked to the NG-FMS/MMS. When a valid 4DT cannot be identified among the NG-FMS/NG-MMS intents, the 4-PNV calculates a viable set family of optimal 4DT based on the performance weightings defined as part of an automationassisted Collaborative Decision-Making (CDM) process, and uplinks it to the aircraft upon ATCO's approval. The NGFMS/MMS then analyses the received 4DT set, assessing the compliance with aircraft performance and operational envelope, and presents them to the flight crew/remote pilots in enhanced Human-Machine Interface and Interactions $\left(\mathrm{HMI}^{2}\right)$ formats. After reviewing and selecting the preferred solution, the flight crew/remote pilots issue a confirmation to the 4-PNV. The increased automation relieves the human operators from repetitive low-level tasks and, thanks to the increased efficiency, enables more efficient traffic flows and environmentallyoptimised flight paths.

\section{4-PNV System Requirements}

The following requirements were set for the conceptual design of the 4-PNV system, featuring "single attempt" negotiation of optimized 4DT intents [6]:

1. The system shall consistently identify conflict-free 4DT solutions for each traffic within its jurisdiction;

2. The automated 4DT management algorithms shall fulfill the timeframe requirements for online tactical operations, as discussed in [7];

3. The system shall be able to negotiate and validate the 4DT intents of multiple aircraft, with multiple equipage in dense air traffic conditions;

4. The system shall ultimately reduce the workload of human operators and improve their situational awareness;

5. The system shall pursue the global optimality of air traffic flows in terms of environmental and economic sustainability objectives, rather than the optimality of individual aircraft trajectories.

\section{System Architecture}

The online processes are distributed across the NG-FMS/NGMMS, the Airline Operations Centers (AOC), the Air Navigation Service Providers (ANSP), the regional Air Traffic Flow
Management (ATFM) units and the ground-based ATM infrastructure. The ground-based infrastructure is distributed among a number of Area Control Centers (ACC) and secondary facilities. Each center/facility $i$ is typically tasked with the tactical control of a number of airspace sectors $j$, as well as some level of strategic flow management and airspace management duties. Fig. 2 depicts a schematic of the distributed architecture including the 4-PNV system, the NG-ADL, the System-Wide Information Management (SWIM) and NG-FMS/NG-MMS.

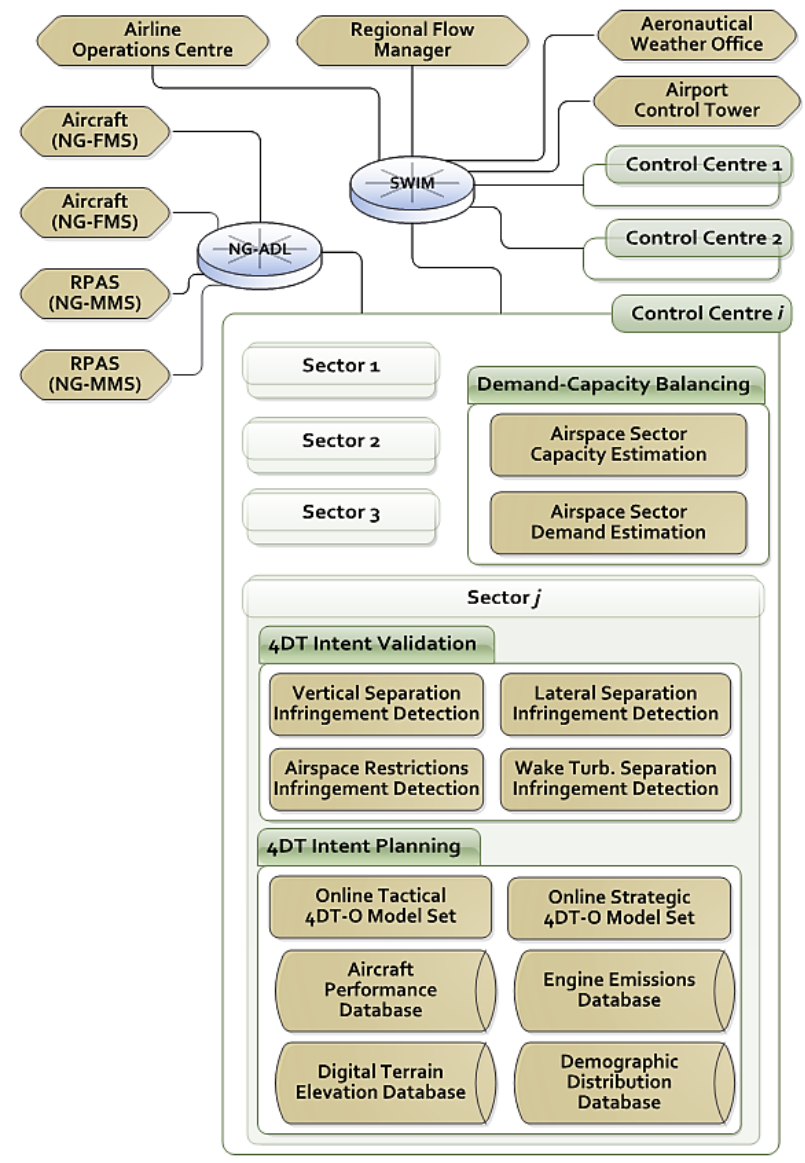

Figure 2. Architecture of the distributed ATM framework integrating the novel CNS+A systems.

\section{D Trajectory Optimization}

The real-time multi-objective trajectory optimization algorithms implemented in the 4-PNV system adopt the same models implemented in the NG-FMS/MMS. The aircraft dynamic parameters are shared between the NG-FMS/MMS and the 4PNV through the NG-ADL, along with the relevant aircraft states, to ensure synchronization and mathematical consistency. The adopted three degrees of freedom (3DOF) point-mass dynamics model with variable mass is: 


$$
\dot{\boldsymbol{x}}=\left\{\begin{array}{l}
\dot{v}=\frac{\tau \cdot T_{C L}-D}{m}-g \sin \gamma \\
\dot{\gamma}=\frac{g}{v} \cdot(N \cos \mu-\cos \gamma) \\
\dot{\chi}=\frac{g}{v} \cdot \frac{N \sin \mu}{\cos \gamma} \\
\dot{\phi}=\frac{v \cos \gamma \sin \chi+v_{w_{\phi}}}{R_{E}+z} \\
\dot{\lambda}=\frac{v \cos \gamma \cos \chi+v_{w_{\lambda}}}{\left(R_{E}+z\right) \cos \phi} \\
\dot{z}=v \sin \gamma+v_{w_{z}} \\
\dot{m}=-F F
\end{array}\right.
$$

Where $v$ is the true airspeed, $\boldsymbol{v}_{\boldsymbol{w}}$ is the wind velocity vector, $\gamma$ is the flight path angle, $\chi$ is the track angle, $m$ is the aircraft mass, $\phi \lambda$ and $z$ are respectively the geodetic latitude, longitude and altitude, $g$ is the gravity acceleration, $R_{E}$ is the geodetic Earth radius, $D$ is the aircraft drag, $T_{C L}$ is the maximum climb thrust. The control variables are $\boldsymbol{u}=\{N, \tau, \mu\}$, which respectively represent the load factor, the throttle and the bank angle. The drag is calculated with the conventional parabolic approximation as:

$$
D=\frac{1}{2} \rho v^{2} S C_{D 0}+\frac{2 C_{D 2} m^{2} g^{2}}{\rho v^{2} S}
$$

Where $\rho$ is the local air density, and $S, C_{D 0}, C_{D 2}$ are respectively the aerodynamic reference surface and the two parabolic drag coefficients that can be obtained by the Eurocontrol's Base of Aircraft Data (BADA) database [10]. The drag coefficient increases to account for flaps and landing gear are also available. Adopting the formulation from BADA, the maximum climb thrust and the fuel flow of a turbofan engine are calculated as [10]:

$$
\begin{gathered}
T_{C L}=C_{T 1} \cdot\left(1-\frac{H_{P}}{C_{T 2}}+C_{T 3} \cdot H_{P}^{2}\right) \\
\cdot\left[1-C_{T 5} \cdot\left(\Delta T-C_{T 4}\right)\right] \\
F F=\max \left[\tau C_{f 1}\left(1+\frac{v_{T A S}}{C_{f 2}}\right), C_{f 3}\left(1-\frac{H_{P}}{C_{f 4}}\right)\right]
\end{gathered}
$$

Where $C_{T 1} \ldots C_{T 5}, C_{f 1} \ldots C_{f 4}$ are the thrust and fuel flow coefficients from the BADA empirical models [10]. The emission of a generic Gaseous Pollutant (GP) is generically modelled as:

$$
G P=\int_{t_{0}}^{t_{f}} E I_{G P} \cdot F F d t \quad[\mathrm{Kg}]
$$

The specific carbon monoxide $(\mathrm{CO})$ and unburned hydrocarbon (HC) emission indexes are empirically modelled as:

$$
E I_{C O / H C}=c_{1}+\exp \left(-c_{2} \tau+c_{3}\right)[\mathrm{g} / \mathrm{Kg}]
$$

Similarly, the nitrogen oxides $\left(\mathrm{NO}_{\mathrm{X}}\right)$ emission index is empirically modelled as:

$$
E I_{N O_{X}}=c_{1} \tau^{2}+c_{2} \tau+c_{3}[\mathrm{~g} / \mathrm{Kg}]
$$

Eq. 6 and 7 are nonlinear fit expressions of the data available in the ICAO Emissions Databank [11]. The generic coefficients providing an accurate fit for most of the currently flown turbofan engines are $c_{1}=0.556 ; c_{2}=10.21 ; c_{3}=4.068$ for $E I_{C O}$, $c_{1}=0.083, c_{2}=13.2 ; c_{3}=1.967$ for $E I_{H C}$ and $c_{1}=7.32 ; c_{2}=$ $17.07 ; c_{3}=3.53$ for $E I_{N O x}$. Linearized models can be derived to enhance computational performance when required. Individual performance indexes $J_{i}$ are obtained associated with all the environmental impacts. In particular, the performance indexes relative to gaseous emissions as well as to the perceived noise can be obtained with cost functions of the Lagrange form (i.e. integral costs) similarly to eq. 5, whilst performance indexes associated with carbon dioxide, fuel consumption and other cumulative costs can be calculated with cost functions of the Mayer form (i.e. terminal costs). The mitigation of contrailinduced radiative forcing is performed by integrating the dedicated contrail model described in $[12,13]$. The performance index reflecting the penalties associated with the transit through a dynamic penalty volume can be associated with the following generalised cost function [7]:

$$
J_{i}=\sum_{j=1}^{n_{\text {sect }}} \int\left[P F_{i, j} \cdot\left(\boldsymbol{x}(t) \cap S_{j}(t)\right)\right] d t
$$

where $P F_{i, j}$ are the penalty factors associated to the $\mathrm{i}^{\text {th }}$ objective in the $\mathrm{j}^{\text {th }}$ penalty volume, and $S_{j}(t)$ is the $\mathrm{j}^{\text {th }}$ dynamic penalty volume.

\section{DT Optimization Algorithm}

Since computational times are a crucial aspect in the 4DT planning algorithm development, an a priori articulation of preference involving the weighted sum of the various performance indexes $J_{i}$ associated with the various emissions and environmental/economic impacts is employed to combine the multiple conflicting operational, economic and environmental objectives. The numerical algorithm for the solution of the optimal control problem with respect of the resulting combined objective is represented in Fig. 3. The currently employed 4DT optimization algorithm is based on direct solution methods of the global orthogonal collocation category, which are arguably the most attractive methods currently available in terms of computational speed for the iterative solution of nonlinear optimization problems [14]. Additionally, to further enhance the algorithm stability and convergence performances, path constraints and boundary conditions are automatically strengthened on all state and control variables to restrict the search domain as much as feasible. Notwithstanding, the significant nonlinearities present in the incorporated models (aircraft dynamics, weather, etc.) produce highly nonconvex search domains that severely affect iterative convergence methods. These limitations can be overcome with the adoption of a variety of heuristic strategies in a customised solution algorithm. Different hybrid optimisers were proposed, either with a pattern search, an evolutionary algorithm or other heuristic strategies to attain the global optimality [15-25]. 


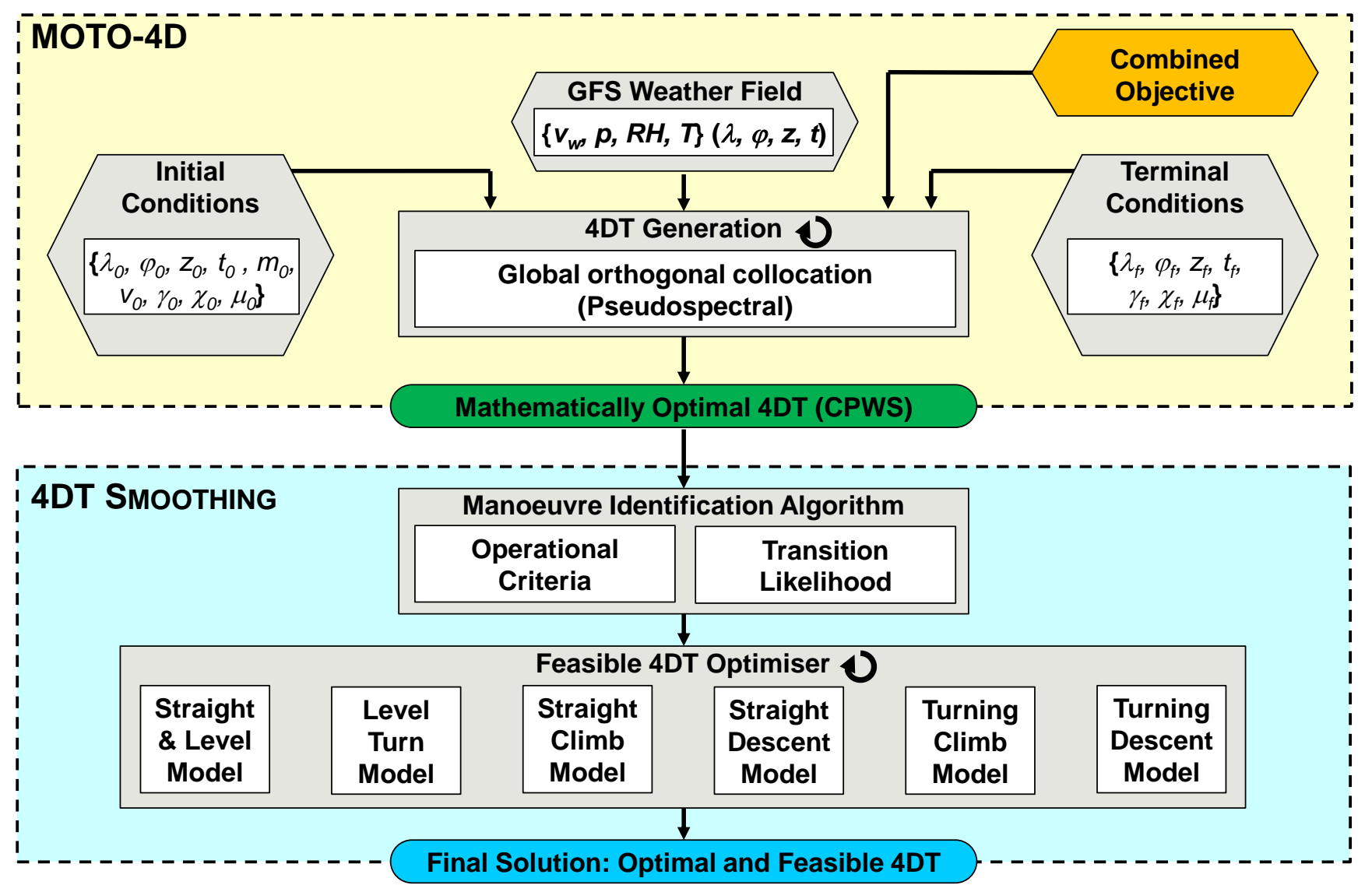

Figure 3. Block diagram of the 4DT optimisation algorithm.

In our 4DT optimiser, a mathematically optimal 4DT is generated by the numerical solution algorithm. This 4DT is a discretised version of a Continuous/Piecewise Smooth (CPWS) curve, which in general may not be flyable by human pilots nor by conventional Automatic Flight Control Systems (AFCS), as it includes transition manoeuvres involving multiple simultaneous variations in the control inputs. Moreover, the discretised CPWS often consists of a very high number of 4D waypoints, which would have unacceptable impacts on the NG.ADL bandwidth usage. Therefore, a post-processing stage is introduced, which employs manoeuvre identification algorithms to segment the trajectory in feasible flight legs, including straight and level flight, straight climbs and descents, level turns, and climbing/descending turns. The final result is a concisely described 4DT consisting of feasible flight segments.

\section{Intent Negotiation and Validation Loops}

A single-attempt negotiation is required in order for the 4-PNV to meet the online tactical rerouting requirements. A 4DT negotiation protocol was developed in EUROCONTROL's DOC 97-70-14 [1] and can be adopted to iteratively generate an optimal flight path, taking all constraints into consideration. Our customized negotiation loops were introduced in [6]. The shared trajectory intents include the aircraft's unique identification and model, the wake-turbulence category, and the vector of 4DT waypoints in the prescribed format. The ground-based 4-PNV system is the protagonist of the strategic online scenario as it retains a continuously updated global situational awareness, thanks to the ground network and to own predictions algorithms. Uncertainties, such as long-term forecast weather cells, or capacity restrictions to airport or to airspace sectors, prompt the 4-

Page 4 of 6
PNV to initiate a strategic re-planning and negotiation by up-linking new constraints to the NG-FMS/NG-MMS, which are then incorporated as part of the on-board 4DT optimization. Alternatively, the 4-PNV may compute optimal 4DT and uplink them for validation by the aircraft. If, after on-board evaluations performances/constraint violations exist (e.g. turn radius, climb rate), the aircraft downlinks a rejection message together with a new intent to the 4-PNV for validation. Multiple negotiation loops are allowed in the strategic online scenario but minimized thanks to the availability of multiple 4DT intents for each aircraft. If for particular reasons the newly introduced constraints cannot be satisfied, then the negotiation may still be pursued through direct human intervention. In the tactical online scenario, either the NG-FMS or the 4-PNV may initiate 4DT intent negotiations. The 4-PNV will act mainly as the centralized decision maker. The NG-FMS may initiate the trajectory negotiation due to locally detected weather phenomena, aircraft performance degradation, equipment failures or on-board emergency situations. Other maneuver-related factors such as inefficient heading changes, and unachievable climb/descent rates and altitudes due to the actual aircraft weight may also be causes of negotiation. In the tactical online scenario, a "single-loop" negotiation is ultimately pursued due to the reduced time and stringent traffic management commitments. Similarly to the strategic online, if a solution cannot be obtained through trajectory negotiation then ATCO's and pilots' direct intervention will be considered. In order to be adopted for both strategic online and tactical online contexts, with a reference time horizon of 5 minutes, it is assumed that the total duration of optimization, negotiation and validation processes must remain under 180 seconds. Trajectories are checked for traffic conflicts and separation from hazardous phenomena. The validation algorithm assesses the lateral and vertical separation criteria and includes a 
simplified wake vortex modeling to assess the longitudinal separation. The Collaborative Decision Making (CDM) is substantially enhanced through the exploitation of semi-automated negotiation schemes.

\section{Simulation and Results}

The sequencing of dense arrival traffic towards a single approach procedure was extensively assumed as a representative case study of online tactical Terminal Manoeuvring Area (TMA) operations. The results of one exemplary simulation run are depicted in Fig. 4. The 4PNV identifies the best arrival sequence among the available options. Longitudinal separation is enforced at the merge-point to ensure sufficient separation upon landing, and to prevent separation infringements in the approach phase itself. The 4-PNV is capable of performing point-merge at any metering point. After the initial intents have been stored in the 4-PNV, the point-merge sequencing algorithm allocates the available time slots accordingly. The assumed minimum longitudinal separation is 4 nautical miles on the approach path for medium category aircraft approaching at 140 knots, therefore the generated time slots are characterized by a 90 160 seconds separation depending on the wake-turbulence categories of two consecutive traffics. Fig. 5 depicts the computed 4DT in the AMAN schedule display format. Waypoints and lines depicted in magenta represent the flyable and concisely-described 4DT consisting of a limited number of fly-by and overfly 4D waypoints, obtained through the smoothing algorithm.

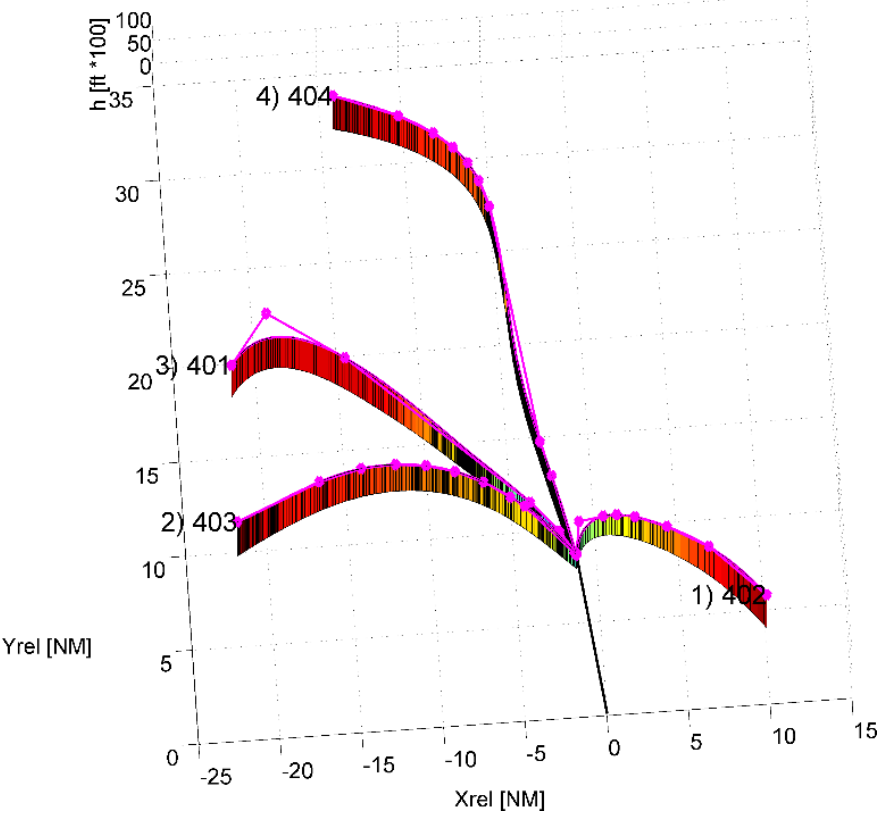

Figure 4. Results of the 4-PNV exploiting the operational 4DT smoothing.

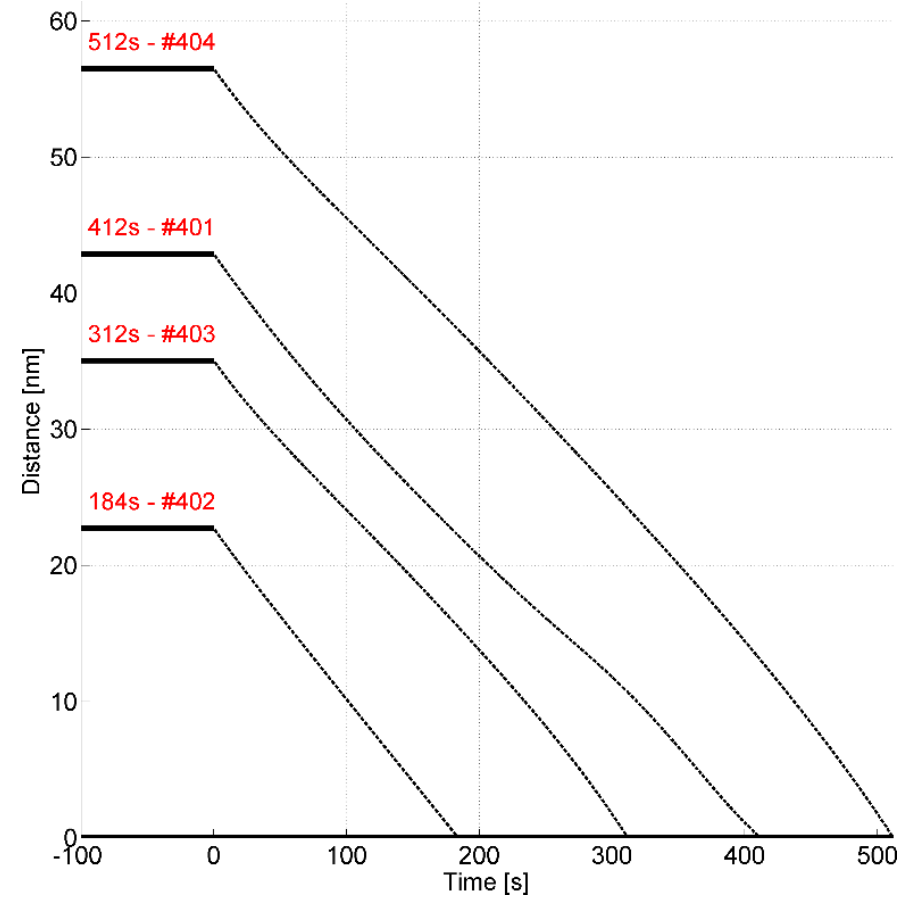

Figure 5. Traffic sequencing represented in an AMAN scheduler plot.

Monte Carlo simulation was performed, resulting in an average of 41 seconds for single newly generated 4DT intents, and consistently less than 60 seconds. The 4DT post-processing allowed to reduce discretised CPWS trajectories of 150 to 450 points into a number of fly-by and overfly 4D waypoints consistently below 20 . These results meet the set design requirements for tactical online data-link negotiation of the 4DT.

\section{Summary}

This paper presented a number of design aspects of a novel 4Dimensional Trajectory Planning, Negotiation and Validation (4PNV) system. The 4-PNV system is intended to operationally deploy the real-time multi-objective optimization of 4-Dimensional Trajectory (4DT) intents in association with the Next Generation Flight Management System (NG-FMS) on board manned aircraft and Mission Management Systems (NG-MMS) on board Remotely Piloted Aircraft Systems (RPAS). The systems are designed to enable planning, negotiation and validation of 4DT intents in the strategic and tactical online Air Traffic Management (ATM) contexts, in the presence of dense air traffic, whenever airspace or air traffic reorganization is required due to tactically changing airspace conditions. Simulation case studies allowed a preliminary assessment of the 4-PNV negotiation and validation models. In high air traffic density conditions, the complete process of NG-FMS/MMS 4DT intent generation, downlink to the 4-PNV and negotiation/validation is performed in less than 180 seconds. These results meet the 3 minutes timeframe assumed for online tactical routing/rerouting tasks and make the approach feasible for quasi-real-time applications. Future research will address the implementation and evaluation of hybrid trajectory optimization algorithms, as well as enhanced algorithms for conflict detection. The concurrent research on safetycritical obstacle avoidance and Detect-and-Avoid (DAA) systems will be highly instrumental in the implementation and assessment of efficient optimization models for 4DT planning [26]. The 4-PNV evolutions will also incorporate various CNS+A integrity monitoring and augmentation strategies currently being researched $[27,28]$. 


\section{References}

[1] I. A. B. Wilson, "Trajectory Negotiation in a Multi-sector Environment", Programme for Harmonised Air Traffic Management Research in EUROCONTROL (PHARE), Bruxelles, Belgium, 1998.

[2] J. E. Robinson III and D. R. Isaacson, "A concurrent sequencing and deconfliction algorithm for terminal area air traffic control", in proceedings of AIAA Guidance, Navigation and Control Conference 2000 (GNC 2000), Denver, CO, USA, 2000. DOI: 10.2514/6.2000-4473

[3] H. Erzberger, "The automated airspace concept", in proceedings of 4th USA/Europe Air Traffic Management Research and Development Seminar (ATM2001), Santa Fe, NM, USA, 2001

[4] E. Mueller, "Experimental Evaluation of an Integrated Datalink and Automation-Based Strategic Trajectory Concept", in proceedings of AIAA Aviation Technology, Integration and Operations conference 2007 (ATIO2007), Belfast, Northern Ireland, 2007. DOI: 10.2514/6.2007-7777

[5] E. Mueller and S. Lozito, "Flight deck procedural guidelines for datalink trajectory negotiation", in proceedings of 8th AIAA Aviation Technology, Integration and Operations Conference (ATIO 2008), Anchorage, AK, 2008. DOI: 10.2514/6.2008-8901

[6] A. Gardi, R. Sabatini, S. Ramasamy, and K. de Ridder, "4Dimensional Trajectory Negotiation and Validation System for the Next Generation Air Traffic Management", in proceedings of AIAA Guidance, Navigation, and Control Conference (GNC 2013), Boston, MA, USA, 2013. DOI: 10.2514/6.2013-4893

[7] A. Gardi, R. Sabatini, S. Ramasamy, and T. Kistan, "Real-Time Trajectory Optimisation Models for Next Generation Air Traffic Management Systems", Applied Mechanics and Materials, vol. 629, pp. 327-332, $2014 . \quad$ DOI: 10.4028/www.scientific.net/AMM.629.327

[8] A. Gardi, R. Sabatini, S. Ramasamy, and M. Marino, "Automated ATM system for 4-dimensional trajectory based operations", in proceedings of 16th Australian International Aerospace Congress (AIAC16), Melbourne, Australia, 2015. DOI: $10.13140 / 2.1 .3635 .0244$

[9] A. Gardi, R. Sabatini, T. Kistan, Y. Lim, and S. Ramasamy, "4Dimensional Trajectory Functionalities for Air Traffic Management Systems", in proceedings of Integrated Communication, Navigation and Surveillance Conference (ICNS 2015), Herndon, VA, USA, 2015. DOI: 10.1109/ICNSURV.2015.7121246

[10] "User Manual for the Base of Aircraft Data (BADA) Revision 3.11", Eurocontrol Experimental Centre (EEC) Technical/Scientific Report No. 13/04/16-01, Brétigny-surOrge, France, 2013.

[11] ICAO. ICAO AIRCRAFT ENGINE EMISSIONS DATABANK [Online]. Available: http://easa.europa.eu/node/15672

[12] Y. Lim, A. Gardi, and R. Sabatini, "Modelling and evaluation of aircraft contrails for 4-dimensional trajectory optimisation", $S A E$ Technical Paper 2015-01-2538, 2015. DOI: 10.4271/2015-012538

[13] Y. Lim, A. Gardi, M. Marino, and R. Sabatini, "Modelling and Evaluation of Persistent Contrail Formation Regions for Offline and Online Strategic Flight Trajectory Planning", in proceedings of International Symposium on Sustainable Aviation (ISSA 2015), Istanbul, Turkey, 2015

[14] A. V. Rao, "Survey of Numerical Methods for Optimal Control", Advances in the Astronautical Sciences, vol. 135, pp. 497-528, 2010
[15] M. P. Hansen, "Tabu Search Multiobjective Optimisation: MOTS", in proceedings of 13th International Conference on Multiple Criteria Decision Making (MCDM '97), Cape Town, South Africa, 1996

[16] J. A. Lennon and E. M. Atkins, "Preference-Based Trajectory Generation", in proceedings of AIAA Infotech@Aerospace 2007 (I@A 2007), Rohnert Park, CA, USA, 2007. DOI: 10.2514/6.2007-2973

[17] J. Krozel, S. Penny, J. Prete, and J. S. B. Mitchell, "Automated Route Generation for Avoiding Deterministic Weather in Transition Airspace", Journal of Guidance, Control, and Dynamics, vol. 30, pp. 144-153, 2007. DOI: 10.2514/1.22970

[18] M. Soler, A. Olivares, and E. Staffetti, "Hybrid Optimal Control Approach to Commercial Aircraft Trajectory Planning", Journal of Guidance, Control, and Dynamics, vol. 33, pp. 985-991, 2010. DOI: $10.2514 / 1.47458$

[19] M. Kamgarpour, V. Dadok, and C. Tomlin, "Trajectory generation for aircraft subject to dynamic weather uncertainty", in proceedings of 49th IEEE Conference on Decision and Control, Atlanta, Georgia, USA, 2010, pp. 2063-2068. DOI: 10.1109/cdc.2010.5717889

[20] H. Pervier, D. Nalianda, R. Espi, V. Sethi, P. Pilidis, D. ZammitMangion, et al., "Application of Genetic Algorithm for Preliminary Trajectory Optimization", SAE International Journal of Aerospace, vol. 4, pp. 973-987, 2011. DOI: 10.4271/2011-01-2594

[21] Q. McEnteggart and J. Whidborne, "A Multiobjective Trajectory Optimisation Method for Planning Environmentally Efficient Trajectories", in proceedings of UKACC International Conference on Control 2012, Cardif, UK, 2012

[22] A. Alonso-Ayuso, L. F. Escudero, and F. J. Martín-Campo, "A mixed $0-1$ nonlinear optimization model and algorithmic approach for the collision avoidance in ATM: Velocity changes through a time horizon", Computers \& Operations Research, vol. 39, pp. 3136-3146, 2012. DOI: 10.1016/j.cor.2012.03.015

[23] C. Tsotskas, T. Kipouros, and M. Savill, "Biobjective optimisation of preliminary aircraft trajectories using TabuSearch", in proceedings of 7th International Conference on Evolutionary Multi-Criterion Optimization (EMO 2013), Sheffield, UK, 2013

[24] N. Yokoyama, "Path Generation Algorithm for Turbulence Avoidance Using Real-Time Optimization", Journal of Guidance, Control, and Dynamics, vol. 36, pp. 250-262, 2013. DOI: $10.2514 / 1.55979$

[25] M. Dobrovinsky and J. Z. Ben-Asher, "Cruise flight throttle optimization by approximate dynamic programming", in proceedings of 29th International Congress of the Aeronautical Sciences (ICAS 2014), 2014

[26] R. Sabatini, A. Gardi, S. Ramasamy, and M. A. Richardson, "A Laser Obstacle Warning and Avoidance System for Manned and Unmanned Aircraft", in proceedings of IEEE Metrology for Aerospace (MetroAeroSpace 2014), Benevento, Italy, 2014, pp. 616-621. DOI: 10.1109/MetroAeroSpace.2014.6865998

[27] R. Sabatini, T. Moore, and C. Hill, "A new avionics-based GNSS integrity augmentation system: Part 1 - Fundamentals", Journal of Navigation, vol. 66, pp. 363-384, 2013. DOI: $10.1017 / \mathrm{S} 0373463313000027$

[28] R. Sabatini, T. Moore, and C. Hill, "A new avionics-based GNSS integrity augmentation system: Part 2 - Integrity flags", Journal of Navigation, vol. 66, pp. 501-522, 2013. DOI: $10.1017 / \mathrm{S} 0373463313000143$ 